\title{
Rare-Earth Free p-Type Filled Skutterudites: Mechanisms for Low Thermal Conductivity and Effects of Fe/Co Ratio on the Band Structure and Charge Transport
}

\author{
Daniel R. Thompson ${ }^{l}$, Chang Liu ${ }^{l}$, Jiong Yang ${ }^{3}$, James R. Salvador ${ }^{* 2}$, Daad B. \\ Haddad $^{2}$, Nicole D. Ellison ${ }^{2}$, Richard A. Waldo ${ }^{2 \dagger}$ \\ Jihui Yang ${ }^{3 *}$
}

1) Optimal CAE

Plymouth, MI 48170

2) Chemical and Materials Systems Laboratory

GM $R \& D$ Center

Warren, MI 48090

3) Materials Science and Engineering Department,

University of Washington

Seattle, WA 98195-2120

$\dagger$ retired

* Corresponding Authors:

James R. Salvador

Email: james.salvador@gm.com

Jihui Yang

Email: jihuiy@uw.edu 


\begin{abstract}
A key driver of cost for a thermoelectric generator is the thermoelectric materials themselves, and the heavy reliance on rare earth (RE) elements as fillers, particularly for p-type formulations, represents a strategic risk. In the present study, we have investigated fully filled p-type skutterudites with nominal compositions $\mathrm{CaFe}_{x} \mathrm{Co}_{4-x} \mathrm{Sb}_{12}(2 \leq x \leq 3.5)$ using both experimental and theoretical methods. High purity samples were successfully synthesized using a combined melt-spinning (MS) and spark plasma sintering (SPS) technique. Structural analysis confirmed phase-pure samples with high filling fraction $(>90 \%)$, homogeneous filler and transition metal distribution, and exceptionally low levels of oxygen contamination. Electrical and thermal transport property measurements revealed large power factors and unexpectedly low thermal conductivities. Electronic band structure calculations found a rapid increase in the density of states at the Fermi level with increasing Fe content. The large power factors observed in this system are attributable to this effect. Maximum $z T$ of 0.9 was achieved in $\mathrm{CaFe}_{3} \mathrm{CoSb}_{12}$ at $773 \mathrm{~K}$. To understand the low lattice thermal conductivity in these compounds, lattice dynamics calculations were performed. The small size of $\mathrm{Ca}$ atoms and their concomitantly weak interaction with the surrounding $\mathrm{Sb}$ atoms result in a small force constant between fillers and the host, giving rise to a heretofore unreported low frequency optical phonon mode. This low frequency mode at $7 \mathrm{meV}$ is in addition to the previously described mode at $17 \mathrm{meV}$ identified by modeling the $\mathrm{Ca}$ as a simple harmonic oscillator. The lower energy phonon mode imparted by $\mathrm{Ca}$ filling results in a comparable lattice thermal conductivity reduction to early lanthanide species such as La.
\end{abstract}




\section{Introduction}

- Skutterudites are an attractive materials system for mid- to high-temperature waste heat recovery applications because their formulations can be modified to obtain either n-type or p-type conduction. The intrinsic nature of their crystal structure allows for the substitution of a variety of cations onto the $2 a$ crystallographic site. [1-18] This substitution and the resulting charge transfer from the electropositive filler atom to the $\mathrm{CoSb}_{3}$ framework leads to n-type materials with carrier concentration that can be controlled through the substitution level of the filler as well as the filler's valence. Filling of the voids and the donation of electrons does not substantially alter the conduction band electronic structure. [19] Due to the triply degenerate nature of the low lying conduction band states excellent power factors $\left(S^{2} / \rho\right.$, where $S$ is the Seebeck coefficient and $\rho$ is the electrical resistivity) in excess of $50 \mu \mathrm{W} / \mathrm{cm} \cdot \mathrm{K}^{2}$ are achievable. Filling the voids has the additional benefit of significantly reducing the total and lattice thermal conductivity. [1-18] The combined effects of power factor increase and total thermal conductivity $(\kappa)$ reduction results in thermoelectric figure of merit, $z \mathrm{~T}$, (where $z \mathrm{~T}=\left(S^{2} / \rho \cdot \kappa\right) \cdot \mathrm{T}$ and $\mathrm{T}$ is the absolute temperature) improvement. Some of the best $z$ T values for n-type skutterudites are in excess of 1.4 at 773 K. [15- 16]

P-type skutterudites can be made by substituting transition metals, such as Fe, that are electron deficient relative to Co onto the $8 c$ site. In general the Fe to Co ratio can be tuned to any particular value provided sufficient filler atoms are available to donate compensating charge to the system. From charge balance considerations higher Fe content leads to a higher filling fraction of the cation. This charge offset results in p-type carriers with concentrations on the order of $10^{20}$ to $10^{21} \mathrm{~cm}^{-3}$. [20-26]

P-type skutterudites currently lag the performance of their n-type counterparts due to the unfavorable valence band (VB) structure of $\mathrm{CoSb}_{3}$ whose band edge is dominated by the $\mathrm{Sb} 5 p$ orbitals. [27, 28] This nondegenerate band is highly dispersed leading to a small density of states and low effective masses. While these features bring about high carrier mobility, beneficial for thermoelectric (TE) performance, the small density of states and lack of degeneracy is unfavorable for $S$. As the Fe content increases, Fe $3 d$ bands play an increasingly 
important role in the valence band edge character. [29] The Fe bands tend to have low dispersion with accompanying large densities of states. This is beneficial for $S$, but detrimental to carrier mobility and therefore the maximum power factors for p-type materials are about $60 \%$ that of their n-type counterparts. [29]

On the other hand, the total and lattice thermal conductivity of p-type skutterudites tend to be significantly lower than those of n-type materials, and therefore $z \mathrm{~T}$ values on the order of 1 to 1.2 at $800 \mathrm{~K}$ are achievable. [23-26] This low thermal conductivity generally comes from having a high filling fraction of heavy rare-earth (RE) metals such as $\mathrm{La}, \mathrm{Ce}, \mathrm{Pr}, \mathrm{Nd}, \mathrm{Eu}$, or $\mathrm{Yb}$. The large masses of these fillers result in low vibrational frequencies via the classical relation $\omega=\sqrt{k / m}$ where $\omega$ is the vibrational frequency and $k$ is the restoring force constant related to the strength of the interactions between the filler and the surrounding Sb-atoms. The value $m$, the mass of the oscillator, is commonly replaced by the atomic mass of the filler. The low frequency resonance vibration caused by the fillers is thought to be beneficial for scattering heat carrying phonons via incoherent processes. [21, 30, 31] However, recent neutron spectroscopic experiments have cast doubt on this mechanism, particularly for highly filled p-type materials. [32] Measurements of $\mathrm{LaFe}_{4} \mathrm{Sb}_{12}$ found that the low frequency vibrational modes of La cause an avoided crossing between the optical and acoustical phonon branches resulting in low dispersion for both. This lattice dynamics feature leads to very low group velocities for these modes and poor overall thermal transport. [32] Both explanations rely on the low vibrational frequencies of the filler atoms, and obtaining such an attribute is usually accomplished by heavy RE metal filling.

The reliance on RE metals as fillers presents a challenge to the broad application of the TE technology in the automotive industry. While RE are, in fact, not all that rare the production of these materials is confined to limited geographical regions which drives pricing instabilities. There are several DOE sponsored programs that are specifically targeted to reduce the reliance on RE metals in permanent magnets since their use is perceived as a strategic risk to the cost effective implementation of alternative energy technologies such as wind power and electric vehicles. [33]

Another challenge of using skutterudites for commercial TE application is the production of high quality materials in large scale, cheaply and with low energy inputs. We draw on the previous development work of the 
permanent magnet community, and identified melt spinning (MS) as a potential low cost route to high volume production of skutterudite materials. Previous studies have shown that a combination of MS and spark plasma sintering (SPS) can be used to obtain phase pure filled-skutterudites by combining the annealing and sintering (consolidation) steps into one thereby reducing both production time and energy inputs. [34-38]

To that end we are interested in investigating non-RE based formulations for $\mathrm{p}$-type skutterudites that can be made with high volume manufacturing processes. Previous work suggests that alkali and alkaline earth (AE) metals can both be used to form fully filled skutterudites of the type $R \mathrm{Fe}_{4} \mathrm{Sb}_{12}(R=\mathrm{Na}, \mathrm{K}, \mathrm{Ca}, \mathrm{Ba}$, and $\mathrm{Sr})$. [39-42] Alkali-metal-filled skutterudites tend to be metallic with poor thermoelectric performance and both $\mathrm{Ba}$ and $\mathrm{Sr}$ are prohibitively expensive particularly at their respective weight fractions in fully filled skutterudites. These considerations led us to investigate the TE properties of $\mathrm{CaFe}_{x} \mathrm{Co}_{4-x} \mathrm{Sb}_{12}$ made by a combined MS and SPS process. While $\mathrm{Ca}$ is light weight and is therefore expected to have a rather high vibrational frequency, it is also of comparable size to $\mathrm{Yb}$ which is known to have a rather weak interaction with $\mathrm{Sb}$ atoms (low $k$ ). [41] As a result $\mathrm{Ca}$ may end up having a lower than expected $\omega$.

Another point of optimization is to position the Fermi level close to both the $\mathrm{Sb} 5 p$ and the Fe $3 d$ bands so that one can take advantage of the higher mobility of the former and the higher effective mass and improved $S$ of the latter. $[29,43]$ We attempt to achieve this delicate balance in the carriers by fixing the Ca content at 1 and varying the Fe content between the nominally charge compensated $x=2$ to the highly doped $x=3.5$. Here we present the TE properties of a series of Ca-filled p-type skutterudites $\mathrm{CaFe}_{x} \mathrm{Co}_{4-\mathrm{x}} \mathrm{Sb}_{12}(x=2,2.5,3$, and 3.5). Through a combined approach of experimentations and first-principles calculations we show that increasing Fe content results in a significant increase in the density of states near the Fermi level. This effectively pins the Fermi level and leads to carriers with low mobility. However, due to the increased density of states large Seebeck coefficients, and power factors that approach $33 \mu \mathrm{W} / \mathrm{cm} \cdot \mathrm{K}^{2}$ at $773 \mathrm{~K}$ are achieved in highly doped samples. The measured power factor is close to the predicted optimal value. [29] The relationship between the electronic band structures we calculated and the resulting electrical transport properties are generalizable to all $\mathrm{Co} / \mathrm{Fe}$ containing p-type skutterudites. Ca-filled materials produced in this study were found to have the lowest thermal conductivity 
of any AE-filled skutterudites consistent with previously published results. [42] Phonon calculations reveal that in addition to the high frequency vibrational mode predicted by harmonic approximations [41] there is a second low frequency phonon mode that can be implicated in the reduction of thermal conductivity by introducing an avoided crossing between low frequency optical and acoustical modes which greatly reduces their group velocities. We show that through a combination of cost effective processing and the selection of an appropriate non-rare-earth filler specie we can produce p-type materials whose TE performance is comparable to RE containing formulations.

Finally, we investigate the effects of co-filling using a combination of $\mathrm{Yb}$ and $\mathrm{Ca}$ in equal atomic amounts for additional thermal conductivity reduction. This combination of fillers was chosen to preserve the electronic band structure and relative doping level. We find that the power factors for $\mathrm{Yb}_{0.45} \mathrm{Ca}_{0.45} \mathrm{Fe}_{3} \mathrm{CoSb}_{12}$ are nearly identical to $\mathrm{CaFe}_{3} \mathrm{CoSb}_{12}$, with no reduction in the thermal conductivity and therefore comparable $z \mathrm{~T}$ values.

\section{Experimental}

\subsection{Sample Synthesis}

Pure Co (Puratronic, 22 mesh powders, 99.995\%), Fe (Alfa Aesar, granules, 99.98\%) and Sb (Strem, bar, $99.999+\%)$ were first inductively melted in a boron nitride (BN) crucible at $1673 \mathrm{~K}$. Subsequently, the stoichiometric amounts of Ca (Alfa Aesar, turnings, 99.9\%), and supplemental Sb, to compensate for the vapor losses during the initial step, were added to the resulting charge and inductively melted again at $1473 \mathrm{~K}$ for 3 min in a $\mathrm{BN}$ crucible that was sealed under $\mathrm{Ar}$ in a fused silica ampule. The ingot was melt-spun under flowing Ar at $1523 \mathrm{~K}$ using a home-built system. The quench rate is controlled by the rotational speed of the chrome plated copper wheel and the ejection pressure. In this study, a tangential wheel speed of $20 \mathrm{~m} / \mathrm{s}$ and a 2.5 psi ejection pressure were used. Ribbon mass yields were greater than $85 \%$.

Ribbons were ground by hand for $5 \mathrm{~min}$ in an Ar filled glove box. Pulverized powders were consolidated using a Dr. Sinter SPS-2040 system under dynamic vacuum of $\sim 2 \mathrm{~Pa}$. The temperature was increased at a rate of $75 \mathrm{~K} / \mathrm{min}$ from room temperature to $833 \mathrm{~K}$ and maintained at this temperature for 20 minutes. A constant uniaxial 
pressure of $50 \mathrm{MPa}$ was applied during the heating and holding steps. After sintering, the sample was allowed to cool to room temperature with the load removed.

\subsection{Characterization Techniques}

\section{Structural and Compositional Analysis.}

Sample density, $d$, was determined by measuring the dimensions and mass of the sintered billets. Compared to the theoretical density of unfilled $\mathrm{CoSb}_{3}\left(7.64 \mathrm{~g} / \mathrm{cm}^{3}\right)$, sample density exceeded $95 \%$ in all cases. Powder x-ray diffraction (PXRD) was used to assess phase identity and purity. Lattice parameters were calculated from the diffraction data using TOPAS software. [44] The actual compositions were determined using electron probe microanalysis (EPMA) by averaging eight randomly selected spots on polished sample surfaces. EPMA was performed using a Cameca SX100 Electron Probe Micro Analyzer at an accelerating voltage of $20 \mathrm{kV}$. The refined lattice parameters, the nominal and EPMA derived compositions and some room temperature transport property values for all samples are listed in Table 1. In all samples the EPMA derived compositions are close to the nominal ones.

\section{Thermoelectric Transport Property Measurements.}

Electrical resistivity $(\rho)$ and carrier concentration $(p)$ were measured from $5 \mathrm{~K}$ to $300 \mathrm{~K}$ with a Linear Research AC resistance bridge and a cryostat equipped with a $5 \mathrm{~T}$ magnet. Seebeck coefficient and thermal conductivity in the same temperature range were obtained with a Quantum Design Physical Property Measurement System (PPMS). Electrical resistivity and Seebeck coefficient from $300 \mathrm{~K}$ to $773 \mathrm{~K}$ were assessed using a Linseis LSR-3 system. High-temperature thermal conductivity was calculated from thermal diffusivity $(D)$, specific heat $\left(C_{p}\right)$ and density using the relation $\kappa=d \cdot D \cdot C_{\mathrm{p}}$. Diffusivity was measured with an Anter Flashline-5000 thermal diffusivity analyzer. Specific heat was measured by differential scanning calorimetry with a Netzsch 404c DSC using the ratio method with a sapphire reference. All Ca-filled samples had similar isobaric specific heat values of $0.23 \mathrm{~J} / \mathrm{g} \cdot \mathrm{K}$ near room temperature and increased nearly linearly to $0.25 \mathrm{~J} / \mathrm{g} \cdot \mathrm{K}$ at $773 \mathrm{~K}$.

\subsection{First-Principles Calculations}


First principles calculations were performed with the Vienna ab-initio simulation package (VASP). [45] A generalized gradient approximation (GGA) functional of the Perdew-Burke-Ernzerhof (PBE) form [46] and projected augmented wave (PAW) method $[47,48]$ were used. Details of the electronic structure calculations for skutterudites can be found elsewhere. [29] Lattice dynamic properties of $R \mathrm{Fe}_{4} \mathrm{Sb}_{12}(R=\mathrm{Ca}, \mathrm{Ba}$, and La) were studied via the frozen phonon method [49], which is implemented in the phonopy package. [50] For the phonon study we constructed a $2 \times 2 \times 2$ supercell of fully relaxed formula units and calculated the Hellmann-Feynman forces using small displacements of $1 \mathrm{pm}$ for each nonequivalent atom in the supercell. We set the convergence criteria to be $10^{-6} \mathrm{eV} / \AA$ for the structural relaxation of the $R \mathrm{Fe}_{4} \mathrm{Sb}_{12}$ unit cell, and $10^{-8} \mathrm{eV}$ for static calculations of the displaced supercells to ensure the accuracy of the phonon results.

\section{Results and Discussion}

\subsection{Compositional and Structural Analysis}

We show the PXRD pattern of one sintered bulk sample with nominal composition $\mathrm{CaFe}_{3} \mathrm{CoSb}_{12}$ in Figure 1 (a). Most reflections are indexable to the skutterudite phase. Several weaker reflections can be seen and were later determined, by EPMA, to be trace amounts of $\mathrm{Co}_{x} \mathrm{Fe}_{1-x} \mathrm{Sb}$ and $\mathrm{Co}_{x} \mathrm{Fe}_{1-x} \mathrm{Sb}_{2}$. Lattice constants derived from the PXRD patterns for all samples investigated are plotted as a function of Fe content in Figure 1 (b), and as can be seen the behavior obeys Vegard's law. As the difference of Ca filler concentration is minor, as indicated in EPMA results shown below, lattice expansion is due to the increased Fe content at the $8 c$ site as Fe has a larger atomic radius than Co.

The constituent elements are homogenously distributed throughout the sample as shown in the EPMA x-ray maps. Figure 2(a) (x-ray maps for $\mathrm{CaFe}_{3} \mathrm{CoSb}_{12}$ ) shows minimal amounts of element concentration differences, particularly for Fe and Co. There are minimal amounts of Ca-containing oxide secondary phases in all samples investigated with nearly homogenous distribution of Ca. We find that in all samples the EPMA derived Ca filling fraction exceeded 90\% (Table 1). The backscattered electron (BSE) images not only confirmed phase homogeneity by showing little color variation and therefore low Z-contrast (atomic number) across the scanned areas, but they also show the fine grain size of the resulting materials. 
To investigate the effects of dual element filling on the thermal transport properties another sample containing $\mathrm{Yb}$ and $\mathrm{Ca}$ in equal proportion with an $\mathrm{Fe}$ to $\mathrm{Co}$ ratio of $3: 1\left(\mathrm{Yb}_{0.45} \mathrm{Ca}_{0.45} \mathrm{CoFe}_{3} \mathrm{Sb}_{12}\right)$ was also prepared. As can be seen in Figure 1(b) the lattice constant of this dual filled sample is nearly identical to that of the Ca-only filled sample with the same $\mathrm{Fe}$ and Co ratio, and reflects the comparable ionic radii of $\mathrm{Ca}^{2+}$ and $\mathrm{Yb}^{2+}$. EPMA found a comparably homogenous distribution of $\mathrm{Fe}, \mathrm{Co}, \mathrm{Ca}$, and $\mathrm{Yb}$ for this sample as well, supported by the BSE and $\mathrm{x}$-ray maps shown in Figure 2 (b) for $\mathrm{Yb}_{0.45} \mathrm{Ca}_{0.45} \mathrm{CoFe}_{3} \mathrm{Sb}_{12}$.

We have demonstrated that $\mathrm{Ca}$-filled and $\mathrm{Ca} / \mathrm{Yb}$-filled skutterudites can be made using a combination of melt spinning and SPS. This processing technique obviates the need for annealing steps and results in phase pure materials with a nearly homogenous distribution of fillers, Fe and Co. MS in conjunction with hot pressing and/or hot deformation has been shown to be an economically viable method for the large scale production of permanent magnet materials and we believe, based on the results presented here, analogous processing methods can be used to make skutterudite-based TE materials which are low cost and durable.

\subsection{Electrical Transport Properties and Electronic Band Structures}

The temperature dependence of $\rho$ for all samples investigated are presented in Figure 3(a). For each composition, $\rho$ increased with temperature, indicating metal-like conduction behavior typical of degenerate semiconductors. With increasing Fe/Co ratio, $\rho$ monotonically decreases due to the electron deficiency of Fe as compared to Co and therefore provides more holes. This assertion is supported by Hall effect measurements presented below. There is a large difference in the $\rho$ values between $\mathrm{CaFe}_{2.5} \mathrm{Co}_{1.5} \mathrm{Sb}_{12}$ and $\mathrm{CaFe}_{3} \mathrm{CoSb}_{12}$ despite the rather small compositional difference between these two samples. At $773 \mathrm{~K}, \rho$ of $\mathrm{CaFe}_{3.5} \mathrm{Co}_{0.5} \mathrm{Sb}_{12}$ is less than half of the value of $\mathrm{CaFe}_{2} \mathrm{Co}_{2} \mathrm{Sb}_{12}$. Despite the difference in measurement systems in the range of $5 \mathrm{~K}$ to $300 \mathrm{~K}$ and 330 K to $775 \mathrm{~K}$; the low-temperature and high-temperature $\rho$ values generally show good agreement.

Seebeck coefficients as a function of temperature are shown in Figure 3(b). Positive $S$ values suggest that all samples have hole dominated electrical conduction. $S$ increases with increasing temperature, for all samples investigated up to $773 \mathrm{~K}$. $\mathrm{CaFe}_{2} \mathrm{Co}_{2} \mathrm{Sb}_{12}$ and $\mathrm{CaFe}_{2.5} \mathrm{Co}_{1.5} \mathrm{Sb}_{12}$ show weaker temperature dependence above $700 \mathrm{~K}$, 
saturating at values close to $+170 \mu \mathrm{V} / \mathrm{K}$. The values of $S$, at any given temperature, decrease as the Fe content increases with the exception of $x=2.0$ and 2.5. In this case $S$ for $x=2.5$ sample is slightly higher than the $x=2.0$. As in the case of $\rho$, this general decrease in $S$ with increasing Fe content is attributable to an increase in $p$ due to Fe's electron deficiency as compared to Co. It is interesting to note that unlike the behavior observed for the compositional dependence of $\rho$, the values of $S$ show a far smaller degree of variation with Fe content. The relationship between $p, S$, and $\rho$ will be discussed in more detail below.

Power factors, plotted in Figure 4, represent the overall electrical contribution to $z T$. As was stated above, hole concentrations likely increase with increasing Fe:Co ratio, causing a decrease in both $S$ and $\rho$. Since the reduction of resistivity is much greater than the degradation of $S, \mathrm{CaFe}_{3.5} \mathrm{Co}_{0.5} \mathrm{Sb}_{12}$ possess the largest power factor with a value in excess of $33 \mu \mathrm{W} / \mathrm{cm} \cdot \mathrm{K}^{2}$ at $773 \mathrm{~K}$ which is slightly higher than that of $\mathrm{CaFe}_{3} \mathrm{CoSb}_{12}$. The values of $\mathrm{CaFe}_{2.5} \mathrm{Co}_{1.5} \mathrm{Sb}_{12}$ and $\mathrm{CaFe}_{2} \mathrm{Co}_{2} \mathrm{Sb}_{12}$ are considerably lower and indicate that in these p-type formulations greater power factors are obtained at higher carrier concentrations. The values we obtained for $S^{2} / \rho$ are equal to those predicted for the optimal doping level in Ca-filled skutterudites. [29]

Hall effect measurements were performed on all Ca-filled samples. The Hall coefficient $\left(R_{H}\right)$ was positive for each formulation confirming that holes are the dominant charge carriers, consistent with the sign of $S$. The carrier concentration as a function of temperature is shown in Figure 5(a). As can be seen the apparent $p$ increases with increasing temperature for each sample, and as we conjectured above, $p$ also increases with increasing Fe content. Values of $p$ were calculated from $R_{H}$ assuming a single carrier type, from a single band with a Hall factor equal to 1 . The $R_{H}$ values for the $x=3$ and 3.5 samples were very small, leading to somewhat higher levels of error in the values due to lower signal. Furthermore there was a significant deviation from the linear dependence between the Hall resistance and applied magnetic field above $1.5 \mathrm{~T}$, particularly at temperatures above $200 \mathrm{~K}$. Therefore $R_{H}$ and $p$ were computed from data taken between -1.5 and $1.5 \mathrm{~T}$ for these samples. The deviation can be understood by considering the electronic band structures, which will be discussed below. The Hall mobility $\left(\mu_{H}\right)$ as a function of temperature is shown in Figure 5(b). Near room temperature all samples have a temperature dependence of $\mu_{H}$ close to $\mathrm{T}^{-3 / 2}$; a value anticipated for carriers scattered predominantly by acoustic phonons. This 
result is in contrast to results presented in Ref. [51] which show that as the carrier concentration increases the temperature dependence of $\mu_{H}$ became weaker and was attributable to a significant increase in the degeneracy of the system resulting in a metallic $\mu_{H}$ temperature dependence of $\mathrm{T}^{-1}$. At lower temperatures, below $50 \mathrm{~K}, \mu_{H}$ is temperature independent and this would suggest that a mix of ionized impurity and acoustical phonon scattering of carriers is operant in this temperature regime. Because of the large differences in $\rho$ and $p$ between the $x \leq 2.5$ and the $x \geq 3.0$ samples we also observe large differences in $\mu_{H}$. The room temperature values of $\mu_{H}$ decrease with increasing Fe content and $p$, but the significant drop between the $x=2.5$ and the $x=3.0$ samples suggests a significant change in the carrier scattering when varying the Fe/Co ratio near these values.

First-principles electronic structures of $\mathrm{CaFe}_{\mathrm{x}} \mathrm{Co}_{4-\mathrm{x}} \mathrm{Sb}_{12}(\mathrm{x}=3.5,3.0,2.5$, and 2.0) were calculated in order to shed light on the variations of electrical transport properties with varying Fe/Co ratios. Figures 6(a)-(c) show the projected band structures onto the transition metals of $\mathrm{CaFe}_{2} \mathrm{Co}_{2} \mathrm{Sb}_{12}$ and $\mathrm{CaFe}_{3.5} \mathrm{Co}_{0.5} \mathrm{Sb}_{12}$ near their Fermi levels. Here the Fermi levels are determined by the experimental $p$ and calculated band structures via semiconductor statistics. By comparing Figures 6(a) and (b), it can be concluded that the Fe bands are much higher in energy than the Co bands by about $0.4 \mathrm{eV}$, consistent with the previous work. [29] These heavy Fe bands pin the Fermi levels, thus the electrical transport properties of our $\mathrm{CaFe}_{\mathrm{x}} \mathrm{Co}_{4-\mathrm{x}} \mathrm{Sb}_{12}$ are mainly determined by the Fe $3 d$ bands, analogous to the behavior found in the Fe-only p-type skutterudites. [29] As the Fe content increases the Fermi level moves deeper into the valence band and crosses more of the heavy Fe bands as shown by comparing Figures 6(a) and (c). Since hole conduction in Fe rich samples is dominated by multiple Fe bands we expect to observe nonlinear behavior between the Hall resistance and the applied magnetic field. This is because the assumption of carriers from a single band breaks down and contributions from bands with differing mobilities leads to the non-linear behavior. We calculated the density of states, $N(E)$ shown in Figure 6(d), and the reduced Fermi level, $\xi$, with respect to the top of the heavy Fe bands. As Fe content, $x$, varies from 2.0 to 3.5, $\xi$ increases from 1.29 to 5.21, see Table 1 . The density of states at the Fermi level, $N\left(E_{F}\right)$, also increases substantially as shown in Figure 6(d), and its energy $(\xi)$ dependence is greater than when the single parabolic band approximation is used. The values for $N\left(E_{F}\right)$ and $\xi$ are listed in Table 1. 
The variations of $N\left(E_{F}\right)$ strongly affect the $S$ and $\mu_{H}$. The Mott equation for degenerated semiconductors suggest that $S$ can be expressed as

$$
S=\frac{\pi^{2}}{3} \frac{k_{B}}{q}\left(k_{B} T\right)\left[\frac{N(E)}{p}+\frac{1}{\mu} \frac{d \mu(E)}{d E}\right]_{E=E_{F}}
$$

where $k_{B}$ and $q$ are the Boltzmann constant and the fundamental charge, respectively. [52, 53] Although $p$ increases with Fe content, the increased $N\left(E_{F}\right)$ compensates some of the Seebeck reductions from the increased $p$. That is why we observed the weak $p$-dependence of the Seebeck coefficients in our samples. In terms of the conductivity, according to our previous work, [43] the scattering rate is proportional to the number of electron states at the Fermi level assuming acoustical phonon scattering is dominant. This causes the room temperature values of $\mu_{H}$ to follow a nearly linear trend with the inverse of $N\left(E_{F}\right)$, as shown in the inset of Figure $6(\mathrm{~d})$. In general, increasing the Fe/Co ratio increases the $\xi$, introduces more Fe bands and causes a rapid increase in $N\left(E_{F}\right)$. The features discussed here are expected to be universal for all p-type skutterudite containing Fe and Co.

\subsection{Thermal Conductivities and Lattice Dynamics}

The temperature dependence of the total $(\kappa)$ and lattice thermal conductivity $\left(\kappa_{\mathcal{L}}\right)$ are shown in Figures 7(a) and 7(b), respectively. At low temperatures they behave as typical crystalline materials with thermal conductivity increasing with temperature up to about $80 \mathrm{~K}$. Above this temperature both $\kappa$ and $\kappa_{L}$ decrease due to typical thermal resistance processes including: grain boundary, point defect and Umklapp processes. The values of the thermal conductivity are quite low, particularly by comparison to other AE-filled p-type skutterudites. [42] Figure 8 shows $\kappa_{L}$ as a function of Fe content for the samples investigated in this study as well as several literature values. [42] The $\kappa_{L}$ in Figure 7(b) and Figure 8 were derived from $\kappa$ using the Wiedemann-Franz relation and a Lorenz number equal to $2.0 \times 10^{-8} \mathrm{~V}^{2} / \mathrm{K}^{2}$. Ca-containing skutterudites have the lowest $\kappa_{L}$ of all alkaline earth-filled skutterudites despite it having the lowest mass and by implication the highest harmonic vibrational frequency. In fact, the $\kappa_{L}$ of the Ca-filled materials are comparable to La- and Pr-filled samples in the $\mathrm{R}_{y} \mathrm{Fe}_{4} \mathrm{Sb}_{12}$ formulations. Figure 8 also demonstrates that there is excellent agreement between the values of $\kappa_{\mathrm{L}}$ for the $\mathrm{CaFe}_{\mathrm{x}} \mathrm{Co}_{4-\mathrm{x}} \mathrm{Sb}_{12}$ 
samples of the present study and those of $\mathrm{CaFe}_{4} \mathrm{Sb}_{12}$ from Ref. [42]. The fact that the materials presented here were prepared using a much different technique than those in Ref. [42] would indicate that the resulting low thermal conductivity observed for the Ca-filled materials is an intrinsic material property and is not a by-product of the preparation method and any resulting variation in the microstructure between the two samples. The authors and others have studied the microstructure of MS skutterudites previously. [37, 38] While the MS quench rate can influence microstructure and $\kappa_{L}$, the most significant influence on $\kappa_{L}$ is the filling fraction achieved. For milled skutterudites Rogl et al. demonstrated that the dependence of transport properties on grain size was less prominent in the p-type materials. [54] Therefore for the present p-type study with achieved filling fractions of Ca greater than 0.9 and values of $\kappa_{L}$ in agreement with the melt annealed $\mathrm{CaFe}_{4} \mathrm{Sb}_{12}$ sample from Ref. [42], microstructural considerations are not necessary to explain the low thermal conductivity.

Lattice thermal conductivity decreases with increasing Fe content at, and above, room temperature. It is unlikely that this reduction is due to point defect scattering as the mass and size difference between Fe and Co is small, and as the Fe content increases the transition metal site actually becomes more ordered. Based on EPMA derived compositions, the Ca content changes very little between the samples and so we do not expect that vacancy filling would play a significant role in $\kappa_{\mathcal{L}}$ reduction either. One possible explanation is the increase in the electron-phonon coupling due to increased $N\left(E_{F}\right)$ with increasing Fe content. From the band structure calculations we found a large increase in $N\left(E_{F}\right)$ with Fe content; additionally the $\mu_{H}$ varies with the inverse of $N\left(E_{F}\right)$, which was attributed to a decrease in the mean free path of the carriers due to an enhancement of the electron-phonon coupling. For thermal conductivity a complimentary effect of increasing phonon scattering from the charge carriers could reduce the thermal conductivity thereby resulting in a decrease in both $\mu_{H}$ and $\kappa_{\mathcal{L}}$. This conclusion needs to be treated cautiously as the differences in the values of $\kappa_{L}$ are small. Further, we do not know the exact value nor the temperature dependence of the Lorenz number we should use to calculate the electronic contribution for $\kappa$. Calculated values of the Lorenz number for $R_{y} \mathrm{Fe}_{3} \mathrm{NiSb}_{12}(R=\mathrm{Ba}, \mathrm{Yb}$, and $\mathrm{Nd})$ varied between $1.73 \times 10^{-8}$ and $1.86 \times 10^{-8} \mathrm{~V}^{2} / \mathrm{K}^{2}$ for samples with carrier concentrations between $1.19 \times 10^{21}$ and $8.5 \times 10^{21} \mathrm{~cm}^{-3}$, respectively. [51] Variation of the Lorenz number within this level does not account for the differences in $\kappa_{L}$ observed in the 
samples here, and therefore we can conclude that the reduction in $\kappa_{\mathcal{L}}$ with increasing Fe content is likely due to increased electron-phonon scattering caused by the increasing $N\left(E_{F}\right)$.

Finally, in the plot of $\kappa_{L}$ vs. T there is an upswing in the magnitude of $\kappa_{L}$ for the $x \leq 2.5$ samples above 600 $\mathrm{K}$. This is attributable to bi-polar thermal conduction, which is commonly observed in more lightly doped p-type skutterudites. At higher temperatures minority carriers (in this case electrons) will increase in concentration due to thermal excitation, and they will contribute to the thermal transport by recombining with the majority holes at the cold side. This recombination liberates the gap energy as transported heat that is an additive effect to the unipolar thermal conductivity accounted for by the Wiedemann-Franz relationship. In the samples with higher Fe content the effects of bi-polar thermal conduction are not as apparent. This is due to their deeper $\xi_{\mathrm{s}}$ and consequently lower minority electron concentrations.

To better understand the trends in $\kappa_{L}$, specifically why Ca-filled materials have the lowest values of all AE-filled skutterudites, lattice dynamics calculations were performed. Figure 9 (a) shows the energy variations in different $R \mathrm{Fe}_{4} \mathrm{Sb}_{12}(R=\mathrm{Ca}, \mathrm{Ba}, \mathrm{La}$, and $\mathrm{Yb})$ with respect to small displacements of the fillers around their equilibrium positions. The data are taken from Ref. [41] and directly lead to $k$. Different Fe/Co ratios have minor effects on the results. The "potential well" is determined by the binding energies between the fillers and the skutterudite host, which can be further characterized by the charge states and ionic sizes of the fillers. [55, 56] Higher charge states and larger ionic sizes cause stronger filler-host interactions. Therefore, $\mathrm{Ca}$ and $\mathrm{Yb}$ have the weakest interactions with the host among all the divalent and trivalent fillers, as shown in Figure 9(a) and Ref. [41]. However, due to its low atomic weight, $\mathrm{Ca}$ has a higher resonant frequency than that of $\mathrm{Ba}$, $\mathrm{Sr}$, or $\mathrm{La}$ [41], implying that the Ca-filled p-type $\mathrm{FeSb}_{3}$ should exhibit higher lattice thermal conductivity than $\mathrm{Ba}, \mathrm{Sr}$, or $\mathrm{La}$ filled skutterudites. Experimental results reported by Qiu et al. and those presented here, contradict this prediction. [41] The reason for the contradiction is that the over-simplified harmonic model used to compute resonance frequencies cannot fully capture the lattice dynamics in filled skutterudites; multiple peaks caused by complex filler-Sb interactions may exist. A similar conclusion was reached for Yb-filled skutterudites. [1] Calculated phonon dispersion curves of $\mathrm{CaFe}_{4} \mathrm{Sb}_{12}, \mathrm{BaFe}_{4} \mathrm{Sb}_{12}$, and $\mathrm{LaFe}_{4} \mathrm{Sb}_{12}$ are presented in Figures 9(b)-(d). Through the 
calculations we discovered that in addition to these previously described high frequency phonons in Ca-filled skutterudites, a lower frequency branch exists in a comparable energetic range to the La modes $(\sim 7 \mathrm{meV})$. This lower frequency mode found for the $\mathrm{Ca}$ filler is lower than that of $\mathrm{Ba}(\sim 8.5 \mathrm{meV})$. The loose bond between $\mathrm{Ca}$ and the host lattice leads to the existence of this secondary low-frequency phonon branch, despite the light weight of $\mathrm{Ca}$ atoms. From the phonon dispersion curves shown in Figure 9 it can be seen that these low frequency modes are very flat, particularly in the region that is midway between the $\Gamma$ and $P$ points and continuing all the way to the zone boundary edge. These flat acoustical and optical modes lead to very low group velocities and ineffective heat transport in this energetic region. Usually, lower frequency acoustical phonons contribute more to lattice thermal conduction; that is why the $\mathrm{Ba}$ atoms, with higher frequency phonons, are less effective in $\kappa_{L}$ reduction compared to $\mathrm{Ca}$ atoms in filled skutterudites. Our phonon calculations are consistent with neutron spectroscopy of $\mathrm{LaFe}_{4} \mathrm{Sb}_{12}$ that also found poorly dispersed phonon bands in the region of 5.5 to $7.3 \mathrm{meV}$, and based on these findings Koza et al. concluded that the $\mathrm{LaFe}_{4} \mathrm{Sb}_{12}$ system has low thermal conductivity due to typical thermal resistive Umklapp processes. [32] We conclude that the low thermal conductivity observed in the Ca-filled materials presented here is due to the same mechanistic reason. The calculations clearly demonstrate the similarity in the phonon dispersion curves between Ca-fillers and La-fillers in the low energy regime. The fact that we observe comparable values for the lattice thermal conductivity between La- and Ca-filled skutterudites further supports this conclusion (Figure 8).

The $z \mathrm{~T}$ curves for all samples investigated are plotted in Figure 10. With increasing Fe content peak $z \mathrm{~T}$ increased from 0.6 at $773 \mathrm{~K}$ for $\mathrm{CaFe}_{2} \mathrm{Co}_{2} \mathrm{Sb}_{12}$ to 0.9 for $\mathrm{CaFe}_{3} \mathrm{CoSb}_{12}$ due, primarily, to the increase in the power factor at higher Fe content. However, the $z \mathrm{~T}$ decreases to 0.8 at $733 \mathrm{~K}$ in $\mathrm{CaFe}_{3.5} \mathrm{Co}_{0.5} \mathrm{Sb}_{12}$ due to an increase in $\kappa$ which offsets the smaller increase in the power factor.

\subsection{Ca/Yb Double-Filled Samples}

A $\mathrm{Ca}$ and $\mathrm{Yb}$ double-filled sample $\left(\mathrm{Yb}_{0.45} \mathrm{Ca}_{0.45} \mathrm{Fe}_{3} \mathrm{CoSb}_{12}\right)$ was prepared to study the effect of one combination of dual element filling on the transport properties. When compared to the single-filled sample with an identical $\mathrm{Fe} / \mathrm{Co}$ ratio, the lattice parameters are very close (Figure 1(b)) due to the comparable ionic radii of $\mathrm{Yb}^{2+}$ 
and $\mathrm{Ca}^{2+}$. As shown in Figure 11, $\rho$ and $S$ of $\mathrm{CaFe}_{3} \mathrm{CoSb}_{12}$ and $\mathrm{Yb}_{0.45} \mathrm{Ca}_{0.45} \mathrm{Fe}_{3} \mathrm{CoSb}_{12}$ overlay each other. The resulting power factor reached $30 \mu \mathrm{V} / \mathrm{cm} \cdot \mathrm{K}^{2}$ at $780 \mathrm{~K}$. The single-filled Ca-containing sample showed temperature independent $\kappa$ from $300 \mathrm{~K}$ to $700 \mathrm{~K}$ with a small upswing above this temperature. The overall $z \mathrm{~T}$ of these two samples are similar. The $z \mathrm{~T}$ of the double-filled is slightly higher between $300 \mathrm{~K}$ and $500 \mathrm{~K}$, yet flattens above $700 \mathrm{~K}$, while the single-filled sample shows an increasing trend and slightly higher $z \mathrm{~T}$ at peak temperature. The difference in the $z \mathrm{~T}$ values between these two samples is statistically insignificant. This result underscores the fact that $R_{y} \mathrm{Fe}_{x} \mathrm{Co}_{4-x} \mathrm{Sb}_{12}$ skutterudites, when $R$ represents fillers with comparable chemistry, will have similar electrical transport properties for a given $p$. Furthermore, we do not observe a significant reduction in $\kappa$ by co-filling because the low vibrational frequency that we report here for Ca is comparable in energy to the normal modes previously reported for Yb-filled skutterudites. Since all these phonon modes have similar character in terms of their low group velocity, the expectation would be that no significant gains in thermal conductivity reduction would be achievable by co-alloying $\mathrm{Ca}$ and $\mathrm{Yb}$ on the filler site.

\section{Summary and Conclusions}

Using a combined MS-SPS technique, we successfully produced calcium-filled p-type skutterudites $\mathrm{CaFe}_{\mathrm{x}} \mathrm{Co}_{4-\mathrm{x}} \mathrm{Sb}_{12}$ with high filling fraction, homogeneous microstructure and low levels of oxide contamination. Its good thermoelectric performance qualifies it as a potential low-cost alternative to the state-of-the-art RE-filled skutterudites. The unusually low lattice thermal conductivity found in these materials was investigated from a theoretical perspective. Combined Hall effect, Seebeck coefficient, and resistivity measurements in conjunction with band structure calculations indicate that as $\mathrm{Fe}$ content increases in the general formula $\mathrm{CaFe}_{x} \mathrm{Co}_{4-x} \mathrm{Sb}_{12}$, the Fe $3 d$ bands begin to play a more important role in electrical conduction, because the Fermi level is being pushed deep into the valence band with increased $p$. The result is a significant increase in the $N\left(E_{F}\right)$ which improves $S$, while the increased carrier concentration decreases $\rho$. These two results act to increase the power factor up to 33 $\mu \mathrm{W} / \mathrm{cm} \cdot \mathrm{K}^{2}$ for $\mathrm{CaFe}_{3.5} \mathrm{Co}_{0.5} \mathrm{Sb}_{12}$. The increased $N\left(E_{F}\right)$ strengthens the electron-phonon coupling causing both the $\mu_{H}$ and $\kappa_{L}$ to decrease with increasing Fe content. The trends are generalizable to all p-type skutterudites with Fe/Co compositions. 
We have found that Ca-filled samples have the lowest $\kappa_{L}$ of any AE-filled skutterudite and that they are comparable to $\mathrm{LaFe}_{4} \mathrm{Sb}_{12}$. First-principles lattice dynamics calculations revealed low frequency modes with poor energy dispersion and low group velocities that have not been reported before. These phonon features lead to low intrinsic thermal conduction.

\section{ACKNOWLEDGMENTS}

This work is supported by General Motors and by the Department of Energy under Award Number DE-FC26-04NT42278. This work is also supported by Oak Ridge National Laboratory managed by the UT Battelle LLC, for the Department of Energy under contract DE-AC05000OR22725. 


\section{REFERENCES}

1. Pei YZ, Chen LD, Zhang W, Shi X, Bai, SQ, Zhao, YZ Meiu ZG, Li XY, Appl. Phys. Lett. 2006; 89: 221107.

2. Pei YZ, Yang J, Chen LD, Zhang W, Salvador JR, Yang J, Appl. Phys. Lett. 2009; 95: 042101.

3. Zhang J, Xu B, Wang LM, Yu D, Liu Z, He J, Tian Y, Appl. Phys. Lett. 2011; 98: 072109.

4. Chen LD, T. Kawahara T, Tang XF, Goto T, Hirai T, Dyck JS, Chen W, Uher C J. Appl. Phys. 2001; $90: 1864$.

5. Zhao XY, Shi X, Chen LD, Zhang WQ, Zhang WB, and Pei YZ, J. Appl. Phys. 2006; 99: 053711.

6. Puyet M, Lenior B, Duscher A, Dehmas M, Stiewe C, Mueller E, J. Appl. Phys. 2004; 95: 4852.

7. Morelli DT, Meisner GP, Chen B, Hu S, Uher C, Phys. Rev. B 1997; 56: 7376.

8. Nolas GS, Cohn JL, Slack GA, Phys. Rev. B 1998; 58: 164.

9. Nolas GS, Kaeser M, Littelton IV RT, Tritt TM, Appl. Phys. Lett. 2000; 77: 1855.

10. Lamberton Jr. GA, Bhattacharya S, Littleton IV RT, Kaeser MA, Yang J, and Nolas GS, Appl. Phys. Lett. 2002; 80:598.

11. Kuznetsov VL, Kuznetsova LA, Rowe DM, J. Phys. Condens. Matter 2003; 15 : 5035.

12. Yang J, Zhang W, Bai SQ, Mei Z, Chen LD Appl. Phys. Lett. 2007; 90: 192111.

13. Bai SQ, Pei YZ, Chen LD, Zhang WQ, Zhao XY, Yang J , Acta Mater. 2009; 57: 3135.

14. Salvador JR, Yang J, Wang H, Shi, J. Appl. Phys. 2010; 107: 043705.

15. Shi X, Kong H, Li CP, Uher C, Yang J, Salvador JR, Wang H, Chen L, Zhang W, Appl. Phys. Lett. 2008; 92 : 182101.

16. Rogl G, Rogl P, Bauer E, Zehetbauer M, Severe Plastic Deformation, in Koumoto K, Mori T (Eds.), Thermoelectric Nanomaterials, Springer, Heidelberg, 2013, 193-254.

17. Shi X, J. Yang J, Salvador JR, Chi MF, Cho JY, Wang H, Bai SQ, Yang J, Zhang WQ, Chen LD, J. Am. Chem. Soc. 2011; 133: 7837.

18. Shi X, Bai S, Xi L, Yang J, Zhang WQ, Chen LD, Yang J, J. Mater. Res.2011; 26: 1745, and references therein.

19. Yang J, Xi L, Zhang W, Chen LD Yang J, J. Electron. Mater. 2009; 38: 1379.

20. Chen B, Xu JH, Uher C, Morelli DT, Meisner GP, Fleurial JP, Calliat T, Borchenvski A Phys. Rev. B 1997; 55: 1476.

21. Sales BC, Mandrus D, Chakoumakos BC, Keppens V, Thompson JR Phys. Rev. B 1997; 56: 15081.

22. Tang XF, Li H, Zhang Q, Niino M, Goto T J. Appl. Phys.2006; 100: 123702. 
23. Tang XF, Zhang, Q, Chen LD, Goto T, Hirai T J. Appl. Phys. 2005; 97: 093712.

24. Zhang L, Grytsiv A, Kerber M, Rogl P, Bauer E, Zehetbauer M, J. Alloys Compd. 2010; 490: 19.

25. Rogl G, Grytsiv A, Bauer E, Rogl P, Zehetbauer M Intermetallics, 2010; 18: 57.

26. Rogl G, Grytsiv A, Rogl P, Bauer E, Zehetbauer M, Intermetallics, 2011; 19: 546.

27. Nordstöm L, Singh DJ Phys. Rev. B 1996; 53: 1103.

28. Sofo JO, Mahan GD, Phys. Rev. B 1998; 58: 15620.

29. Yang J, Qui P, Liu R, Xi L, Zheng S, Zhang W, Chen LD, Singh DJ, Yang J, Phys. Rev. B 2001; 84: 235205.

30. Yang J, Morelli DT, Meisner GP, Chen W, Dyck JS, Uher C Phys. Rev. B 2003; 67: 165207.

31. Yang J, Zhang W, Bai SQ, Mei Z, Chen LD, Appl. Phys Lett. 2007; 90: 19211.

32. Koza MM, Johnson MR, Viennois R, Mutka H, Girard L, Ravot D Nature Mater. 2008; 7: 806.

33. Rare Earth Alternatives in Critical Technologies. Apra-e programs [online]. http://arpa-e.energy.gov/?q=arpa-e-programs/react (accessed October, 2014)

34. Li H, Tang X, Zhang Q J. Elect. Mater. 2009; 38: 1224.

35. Li H, Tang XF, Su X, Zhang Q, Uher C, J. Phys. D: Appl. Phys. 2009; 42: 145409.

36. Jie Q, Zhou J, Dimitrov IK, Li CP, Uher C, Wang H, Porter W, Li Q Mater. Res. Soc. Symp. Proc. 2010 ; 1267: DD-30-3.

37. Tan G, Liu W, Wang S, Yan Y, Li H, Tang XF, Uher C J. Mater. Chem. A 2013; 1: 12657.

38. Salvador JR, Waldo RA, Wong CA, Tessema MM, Brown DN, Miller DJ, Wang H, Wereszczak AA, Cai W, Mater. Sci and Eng. B 2013; 178:1087.

39. Leithe-Jasper A, Schnelle W, Rosner H, Rabis A, Baenitz M, Gippius AA, Morozova EN, Mydosh JA, and Grin Y Phys. Rev. Lett. 2013; 91: 037208.

40. Leithe-Jasper A, Schnelle W, Rosner H, Baenitz M, Rabis A, Gippius AA, Morozova EN, Borrmann H, Burkhardt U, Ramlau R, Schwarz U, Mydosh JA, Grin Y, Ksenofontov V, Reiman S Phys. Rev. B 2004; 70: 214418.

41. Liu R, Yang J, Chen X, Shi X, Chen LD,Uher C, Intermetallics 2010; 19: 1747.

42. Qui PF, Yang J, Liu RH, Shi X, Huang XY, Snyder GJ, Zhang W, Chen LD, J. Appl. Phys. 2011; 109: 063713.

43. Yang J, Liu R, Xi L, Yang J, Zhang W, Chen LD, Appl. Phys. Lett. 2010; 101: 022101.

44. Bruker AXS GmbH. DiffracPlus: TOPAS 4.2 User Manual. Karlsruhe Germany: Bruker AXS, 2009.

45. Kresse G, Furthmüller J Phys. Rev. B 1996; 54:11169. 
46. Perdew JP, Burke K, Ernzerhof M Phys. Rev. Lett. 1996; 77:3865.

47. Blöchl PE, Phys. Rev. B 1994; 50: 17953.

48. Kresse G, Joubert D Phys. Rev. B 1999; 59: 1758.

49. Martin RM, Electronic structure: basic theory and practical methods. Cambridge University Press, 2004.

50. Togo A, Oba F, Tanaka I Phys. Rev. B 2008; 78: 134106.

51. Liu R, Cho JY, Yang J, Zhang W, Chen LD, J. Mater. Sci. and Tech. B, 2014; 30: 1134.

52. Heremans JP, Jovovic V, Toberer ES, Saramat A, Kurosaki K, Charoenphakdee A, Yamanaka S,Snyder GJ, Science 2008; 321: 554.

53. Heremans JP, Wiendlocha B, Chamoire AM, Ener. and Environ. Sci. 2012; 5: 5510.

54. Rogl G, Grytsiv A, Rogl P, Bauer E, Hochenhofer M, Anbalagan R, Mallik RC, Schafler E, Acta Mater. 2014; 76: 434.

55. Chen Z, Yang J, Liu R,Xi X, Zhang W, Yang J J. Elec. Mater. 2013; 42: 2492.

56. Mei ZG, Yang J, Pei YZ, Zhang W, Chen LD, Yang J, Phys. Rev. B 2008; 77: 045202.

57. Dimitrov IK, Manley ME, Shapiro SM, Yang J, Zhang W, Chen LD, Jie Q. Ehler G, Podlesnyak A, Camacho J, Li Q, Phys. Rev. B 2010, 82: 174301. 
Table 1. List of the nominal and EPMA derived compositions, lattice constant $(a)$, density $(d)$ relative to the theoretical density of $\mathrm{Co}_{4} \mathrm{Sb}_{12}$ which is $7.64 \mathrm{~g} / \mathrm{cm}^{3}$, room temperature carrier density $(p)$, resistivity $(\rho)$, Hall mobility $\left(\mu_{\mathrm{H}}\right)$, Seebeck coefficient $(S)$, thermal conductivity $(\kappa)$, reduced Fermi level $(\xi)$, and density of states at the Fermi level $\left(N\left(E_{F}\right)\right)$ for $\mathrm{CaFe}_{\mathrm{x}} \mathrm{Co}_{4-\mathrm{x}} \mathrm{Sb}_{12}, \mathrm{Ca}_{0.45} \mathrm{Yb}_{0.45} \mathrm{Fe}_{3} \mathrm{CoSb}_{12}$.

\begin{tabular}{|c|c|c|c|c|c|c|c|c|c|c|}
\hline Nominal composition & EPMA Composition & $\begin{array}{l}a \\
(\AA)\end{array}$ & $\begin{array}{c}d \\
(\%)\end{array}$ & $\begin{array}{c}p \\
\left(10^{21} \mathrm{~cm}^{-3}\right)\end{array}$ & $\begin{array}{c}\rho \\
(\mathrm{m} \Omega \cdot \mathrm{cm})\end{array}$ & $\begin{array}{c}\mu_{\mathrm{H}} \\
\left(\mathrm{cm}^{2} / \mathrm{V} \cdot \mathrm{s}\right)\end{array}$ & $\begin{array}{c}S \\
(\mu \mathrm{V} / \mathrm{K})\end{array}$ & $\begin{array}{c}\kappa \\
(\mathrm{W} / \mathrm{m} \cdot \mathrm{K})\end{array}$ & $\xi$ & $\begin{array}{c}N\left(E_{F}\right) \\
\left(\mathrm{eV}^{-1} \text { u.c. }^{-1}\right)\end{array}$ \\
\hline $\mathrm{CaFe}_{2} \mathrm{Co}_{2} \mathrm{Sb}_{12}$ & $\mathrm{Ca}_{0.9 ? \pm 0.06} \mathrm{Fe}_{2} \mathrm{Co}_{2} \mathrm{Sb}_{12}$ & 9.116 & 97 & 0.81 & 0.947 & 8.2 & 102 & 1.94 & 1.29 & 14.31 \\
\hline $\mathrm{CaFe}_{2.5} \mathrm{Co}_{1.5} \mathrm{Sb}_{12}$ & $\mathrm{Ca}_{0.90 \pm 0.05} \mathrm{Fe}_{2.5} \mathrm{Co}_{1.5} \mathrm{Sb}_{12}$ & 9.123 & 97 & 1.5 & 0.88 & 4.8 & 105 & 2.61 & 2.16 & 23.83 \\
\hline $\mathrm{CaFe}_{3} \mathrm{CoSb}_{12}$ & $\mathrm{Ca}_{0.94 \pm 0.05} \mathrm{Fe}_{3} \mathrm{CoSb}_{12}$ & 9.138 & 96 & 4.0 & 0.620 & 2.8 & 90 & 2.74 & 4.41 & 41.21 \\
\hline
\end{tabular}




\section{Figure Captions}

Figure 1: (a) XRD patterns for $\mathrm{CaFe}_{3} \mathrm{CoSb}_{12}$. The most intense reflections match the literature pattern of $\mathrm{CoSb}_{3}(\boldsymbol{\nabla})$. As confirmed in EPMA, trace amounts of impurity phases, $\mathrm{Co}_{x} \mathrm{Fe}_{1-x} \mathrm{Sb}(\bullet)$ and $\mathrm{Co}_{x} \mathrm{Fe}_{1-x} \mathrm{Sb}_{2}(\mathbf{O})$, are present. (b) Lattice constants for $\mathrm{CaFe}_{\mathrm{x}} \mathrm{Co}_{4-\mathrm{x}} \mathrm{Sb}_{12}$ and $\mathrm{Yb}_{0.45} \mathrm{Ca}_{0.45} \mathrm{Fe}_{3} \mathrm{CoSb}_{12}$.

Figure 2: Elemental mapping and backscattered electron (BSE) images of (a) $\mathrm{CaFe}_{3} \mathrm{CoSb}_{12}$ and (b) $\mathrm{Yb}_{0.45} \mathrm{Ca}_{0.45} \mathrm{Fe}_{3} \mathrm{CoSb}_{12}$, showing homogeneous element distribution and low oxygen contamination level.

Figure 3. Temperature dependence of (a) the resistivity and (b) the Seebeck coefficient for $\mathrm{CaFe}_{\mathrm{x}} \mathrm{Co}_{4-\mathrm{x}} \mathrm{Sb}_{12}$ $(x=2,2.5,3$, and 3.5).

Figure 4: Temperature dependence of the power factor for $\mathrm{CaFe}_{\mathrm{x}} \mathrm{Co}_{4-\mathrm{x}} \mathrm{Sb}_{12}(x=2,2.5,3$, and 3.5).

Figure 5: Temperature dependence of (a) the carrier concentration ( $p$ ) and (b) Hall mobility $\left(\mu_{H}\right)$ for $\mathrm{CaFe}_{\mathrm{x}} \mathrm{Co}_{4-\mathrm{x}} \mathrm{Sb}_{12}(x=2,2.5,3$, and 3.5).

Figure 6. (a)-(c) The projected band structures (on transition metals) of $\mathrm{CaFe}_{2} \mathrm{Co}_{2} \mathrm{Sb}_{12}$ and $\mathrm{CaFe}_{3.5} \mathrm{Co}_{0.5} \mathrm{Sb}_{12}$. The error bars denote the contributions of the atoms of interest. (d) Density of states of all the samples investigated. The inset shows the relations between the mobilities and density of states at the Fermi levels $N\left(E_{F}\right)$.

Figure 7: Temperature dependence of (a) total and (b) lattice thermal conductivities for $\mathrm{CaFe}_{x} \mathrm{Co}_{4-x} \mathrm{Sb}_{12}$.

Figure 8. Comparison of room temperature lattice thermal conductivity as a function of Fe content for all the samples investigated in this study as well as literature data for $R \mathrm{Fe}_{4} \mathrm{Sb}_{12}(R=\mathrm{Sr}, \mathrm{Ba}, \mathrm{Pr}, \mathrm{La}, \mathrm{Ce}, \mathrm{Eu}, \mathrm{Yb}$, and $\mathrm{Ca}$ ).

Figure 9: (a) The energy difference vs. the small displacements of fillers around their equilibria. (b)-(d) Phonon dispersions of $\mathrm{CaFe}_{4} \mathrm{Sb}_{12}, \mathrm{BaFe}_{4} \mathrm{Sb}_{12}$, and $\mathrm{LaFe}_{4} \mathrm{Sb}_{12}$. The error bars denote the contributions from the filler atoms.

Figure 10: Temperature dependence of $z \mathrm{~T}$ for $\mathrm{CaFe}_{x} \mathrm{Co}_{4-x} \mathrm{Sb}_{12}$.

Figure 11: (a) Electrical resistivity, (b) Seebeck coefficient, (c) thermal conductivity, and (d) zT of single-filled $\mathrm{CaFe}_{3} \mathrm{CoSb}_{12}$ and double-filled $\mathrm{Yb}_{0.45} \mathrm{Ca}_{0.45} \mathrm{Fe}_{3} \mathrm{CoSb}_{12}$. 

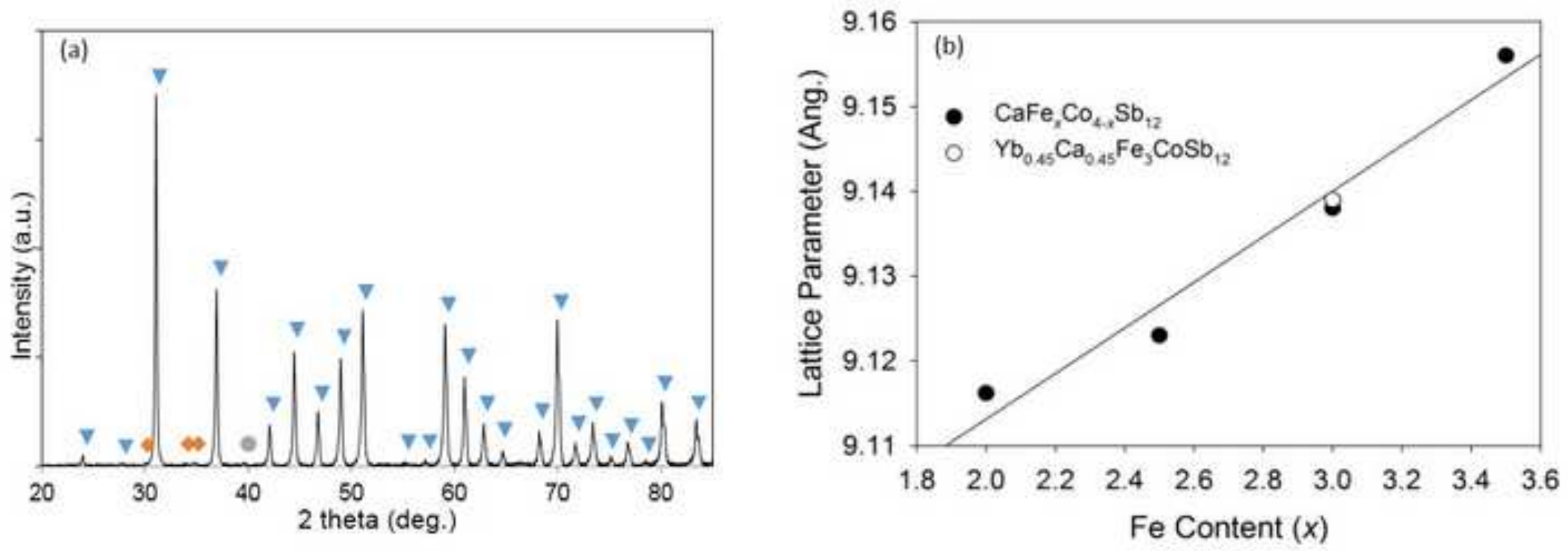
(a)

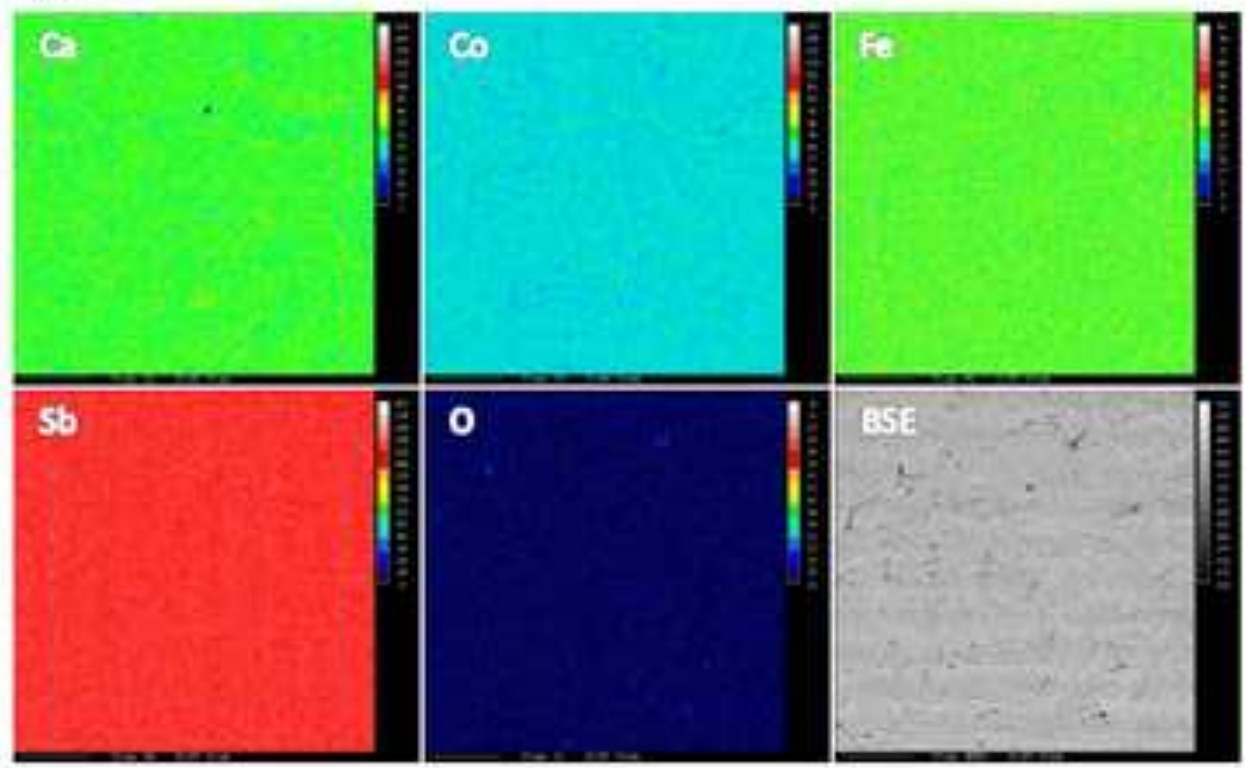

(b)

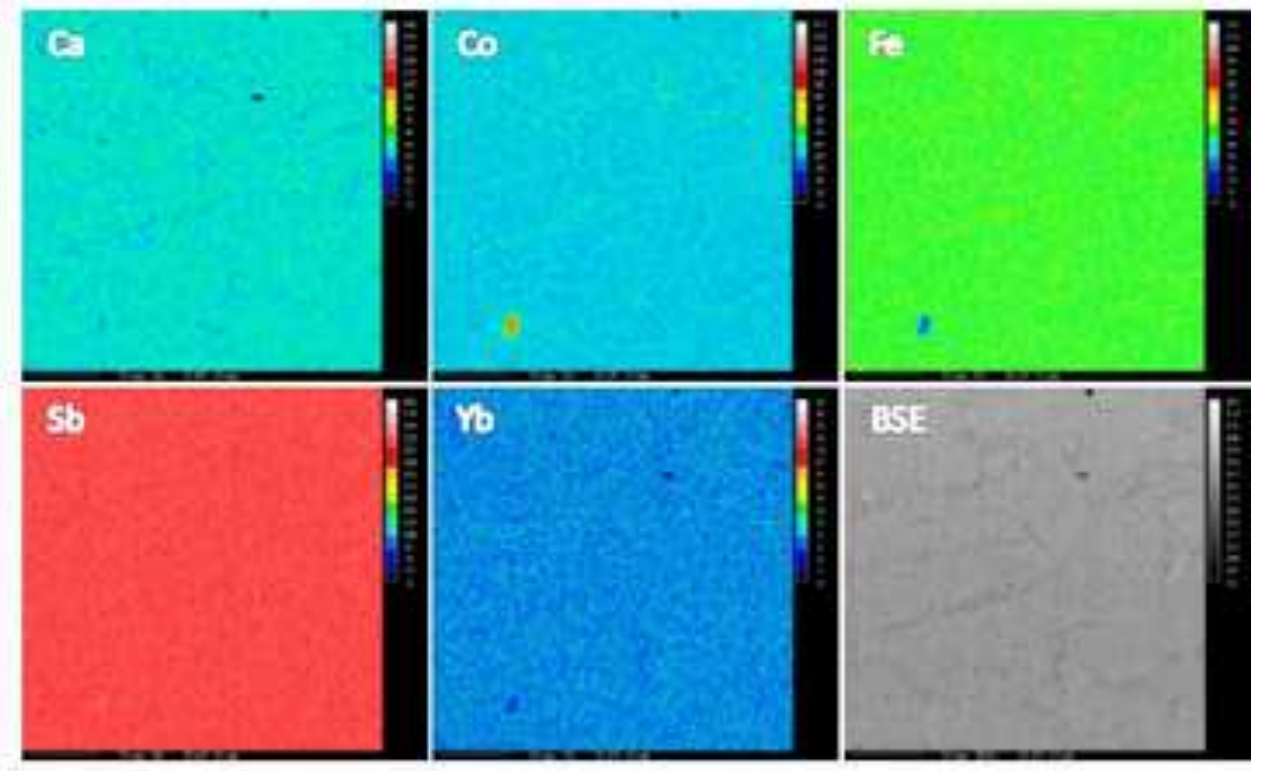




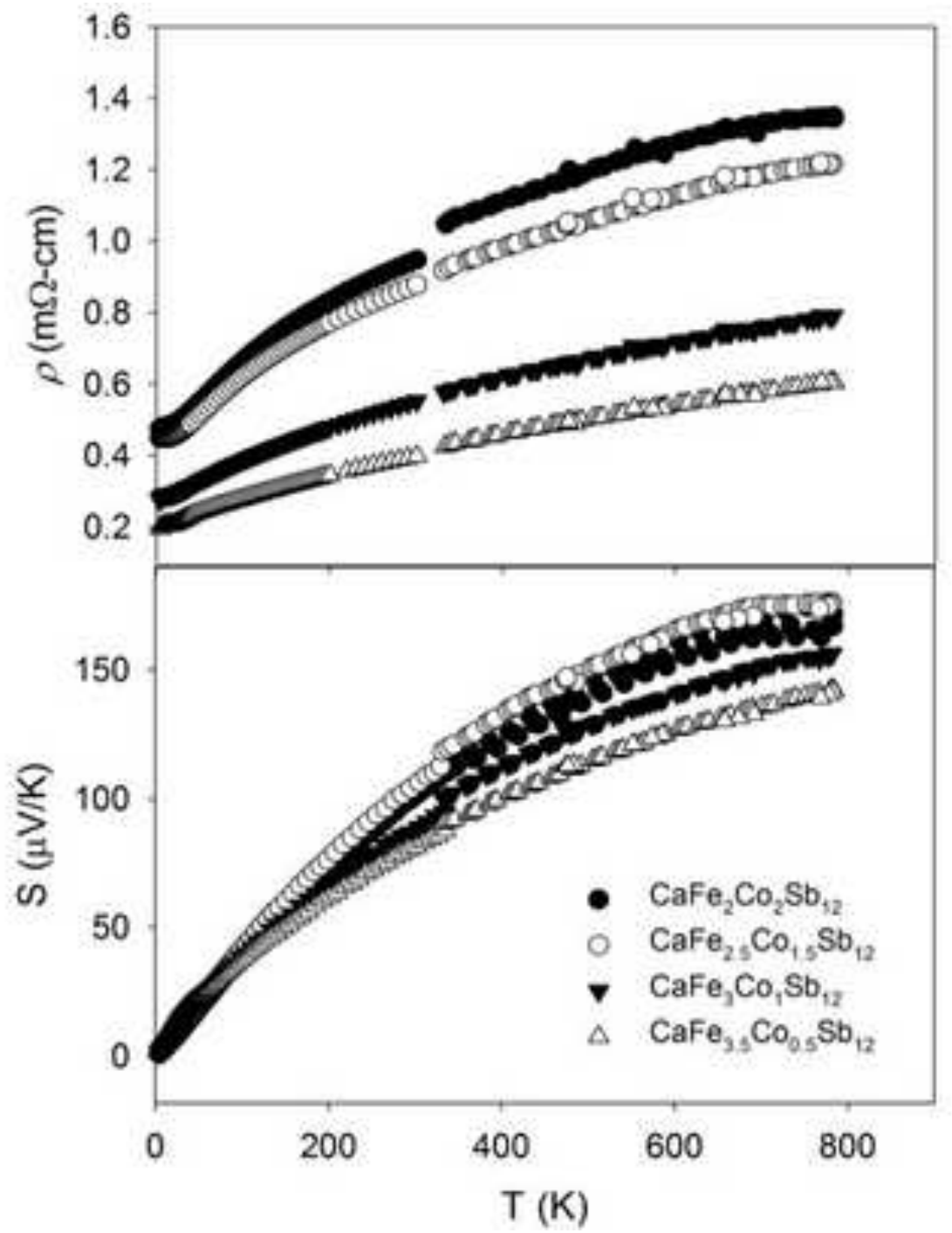




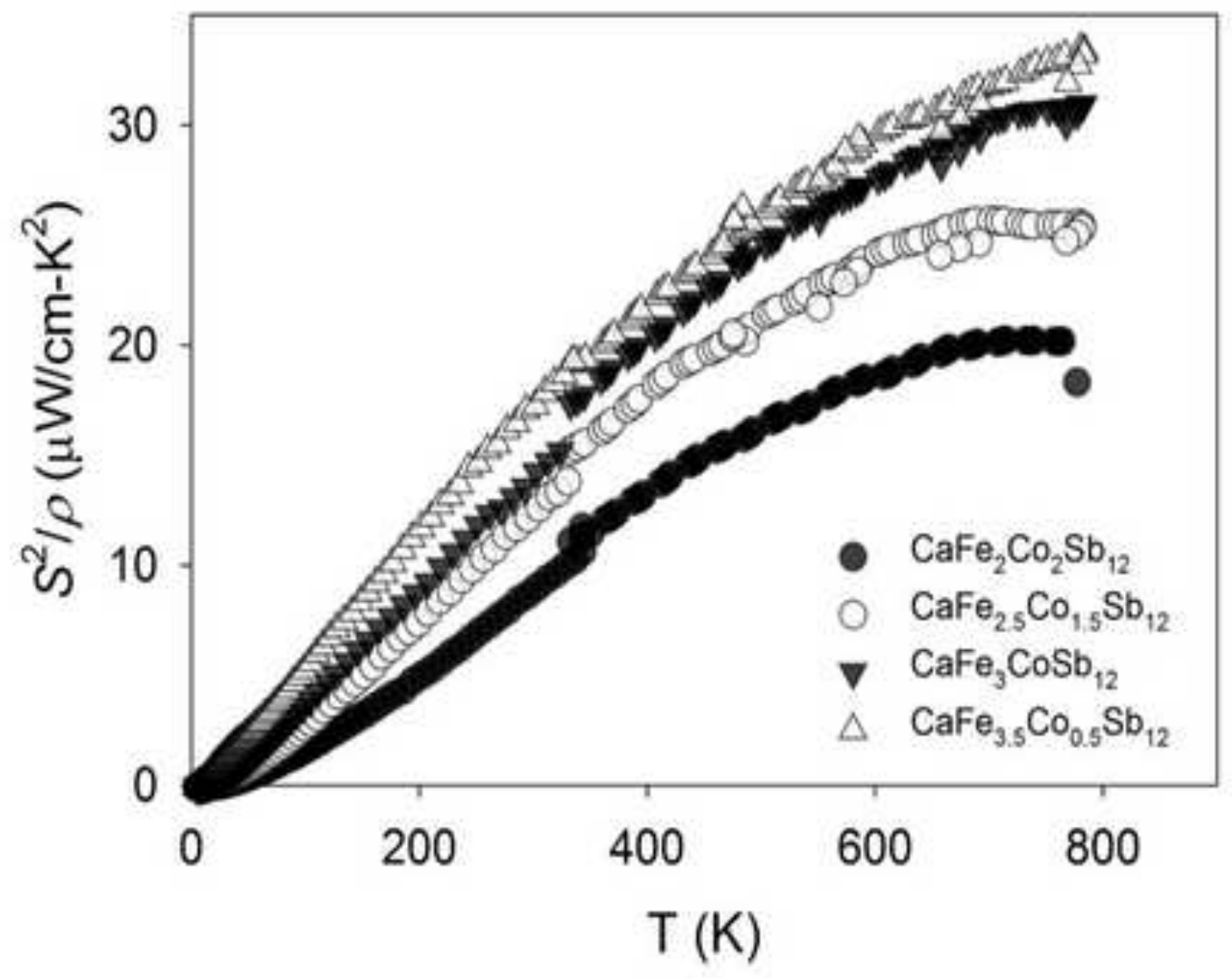



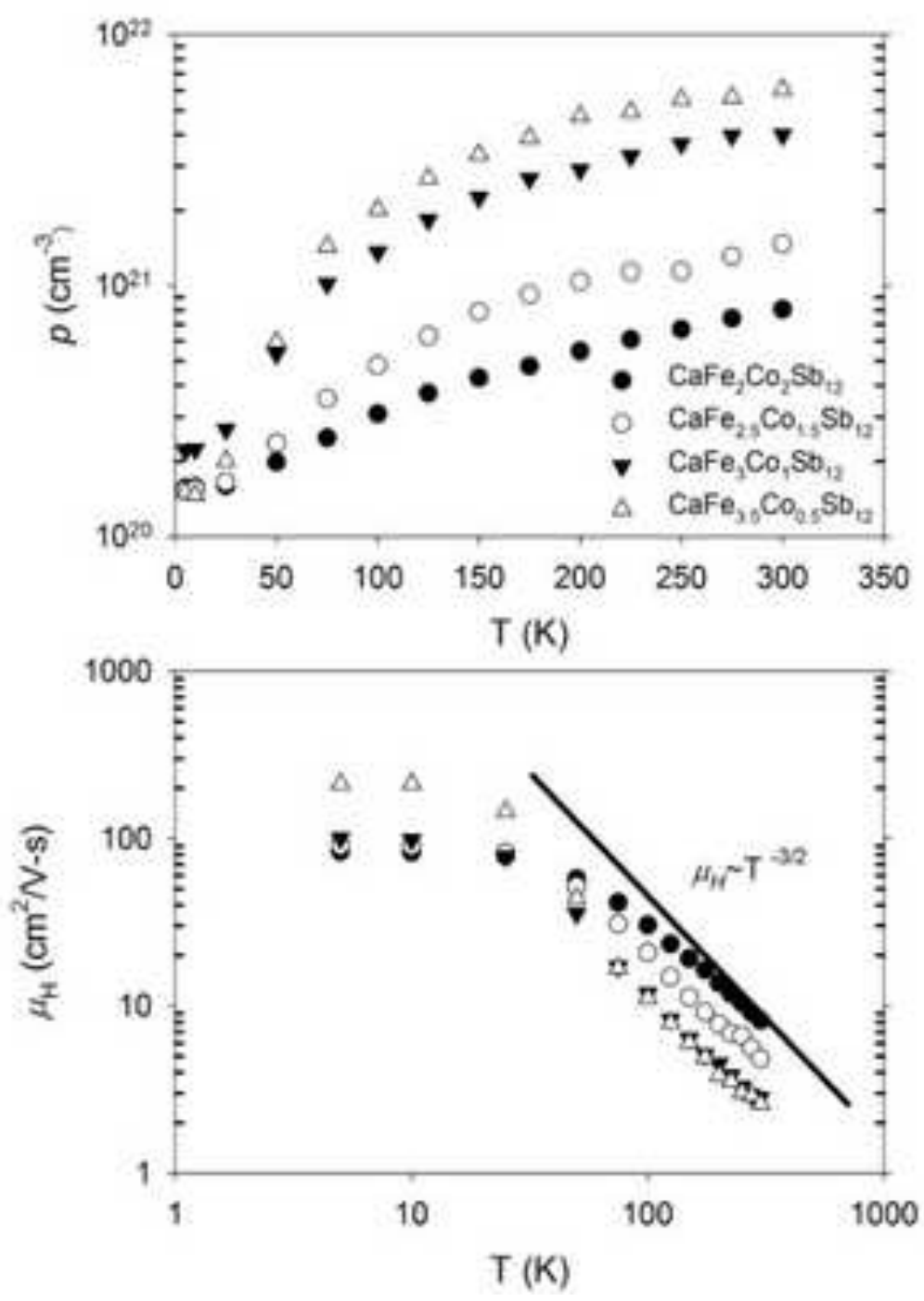

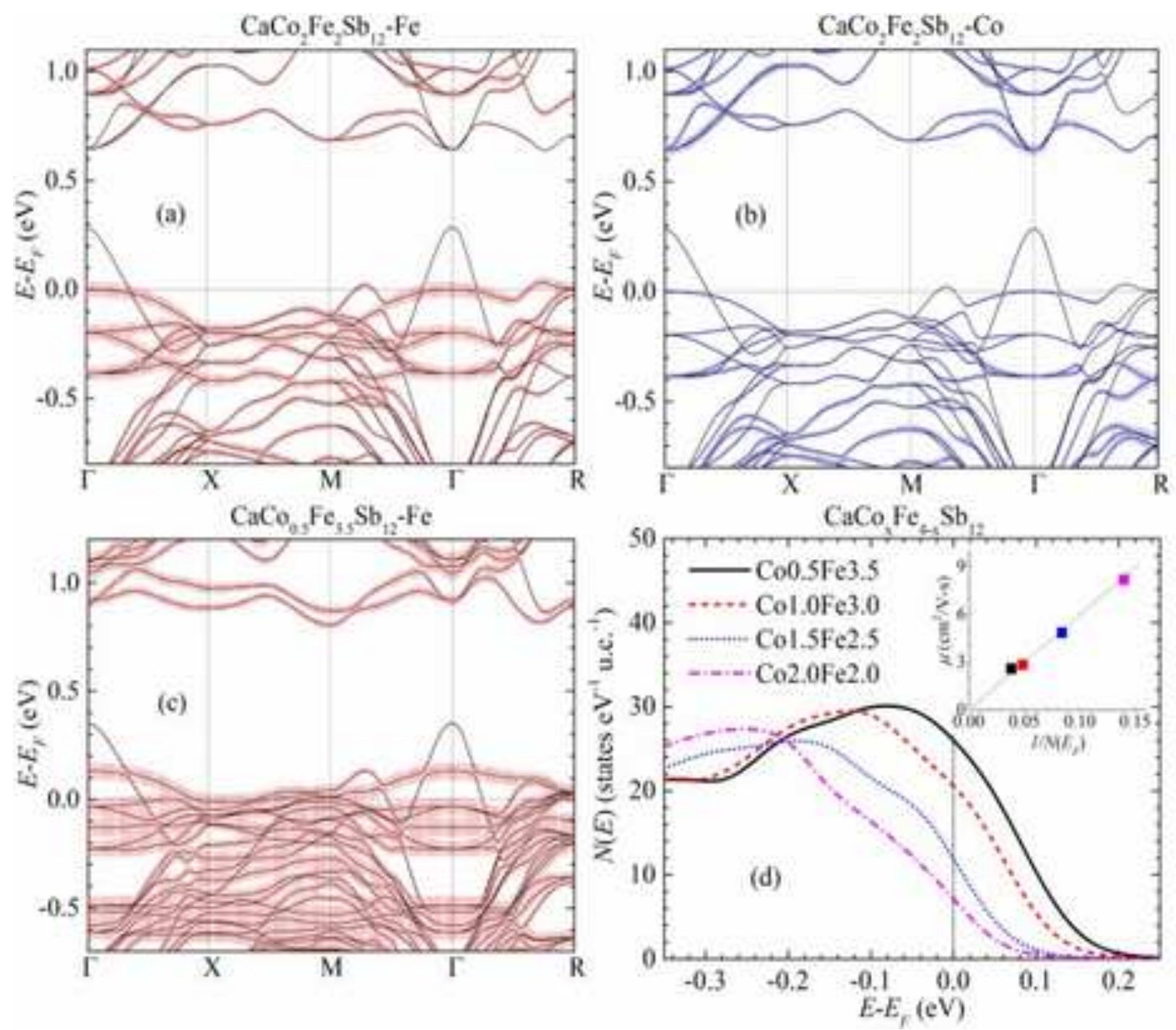


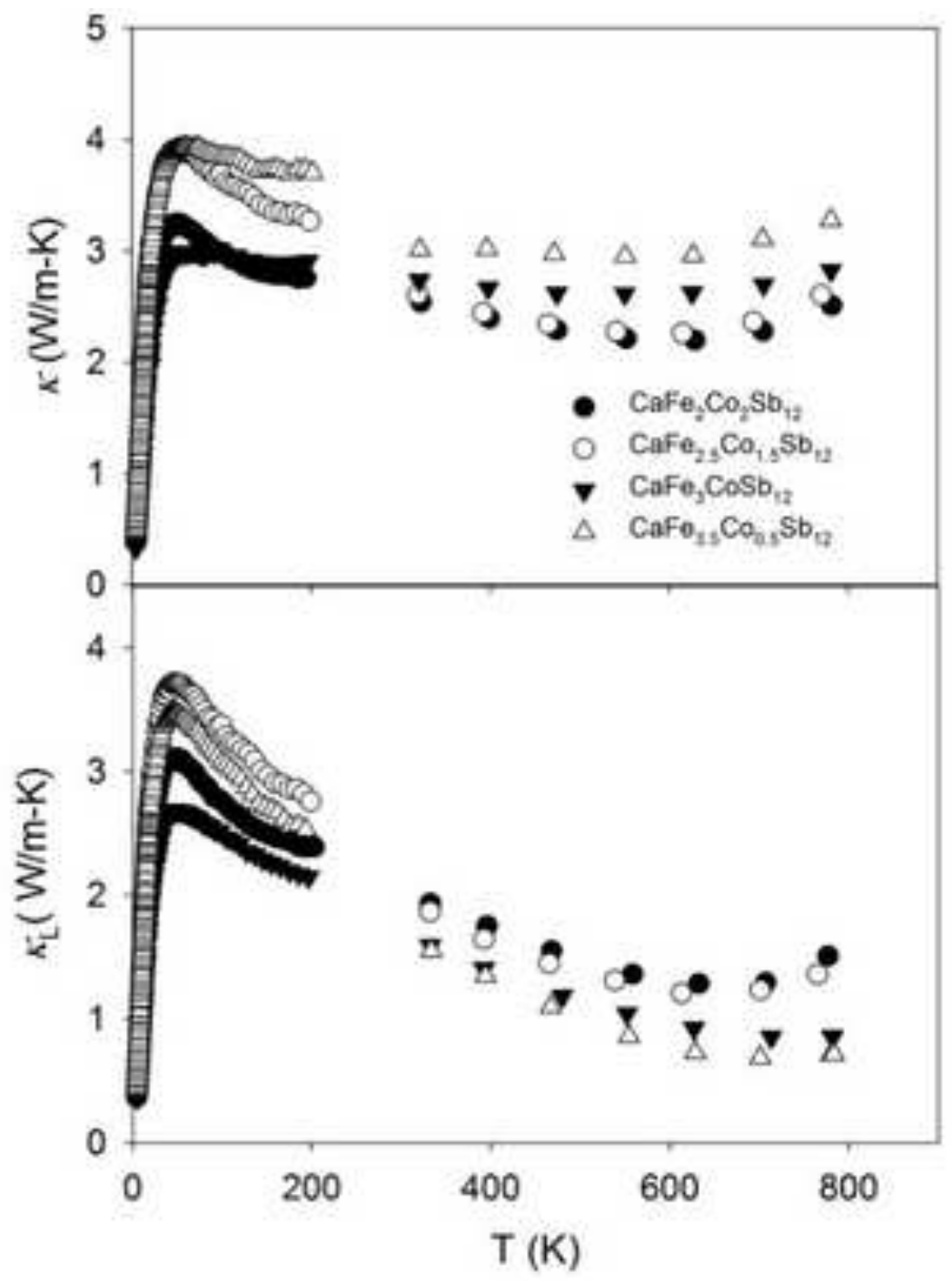




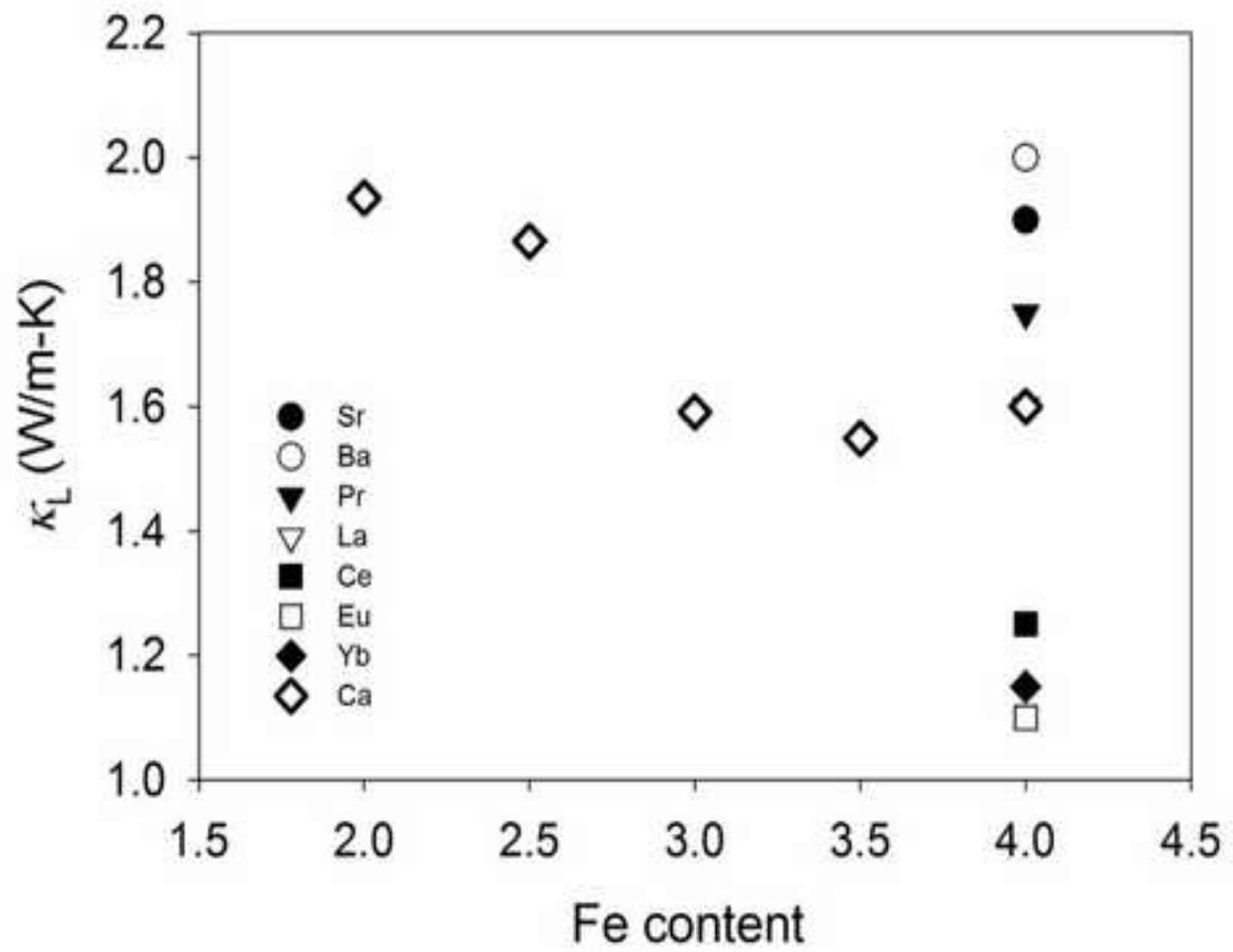



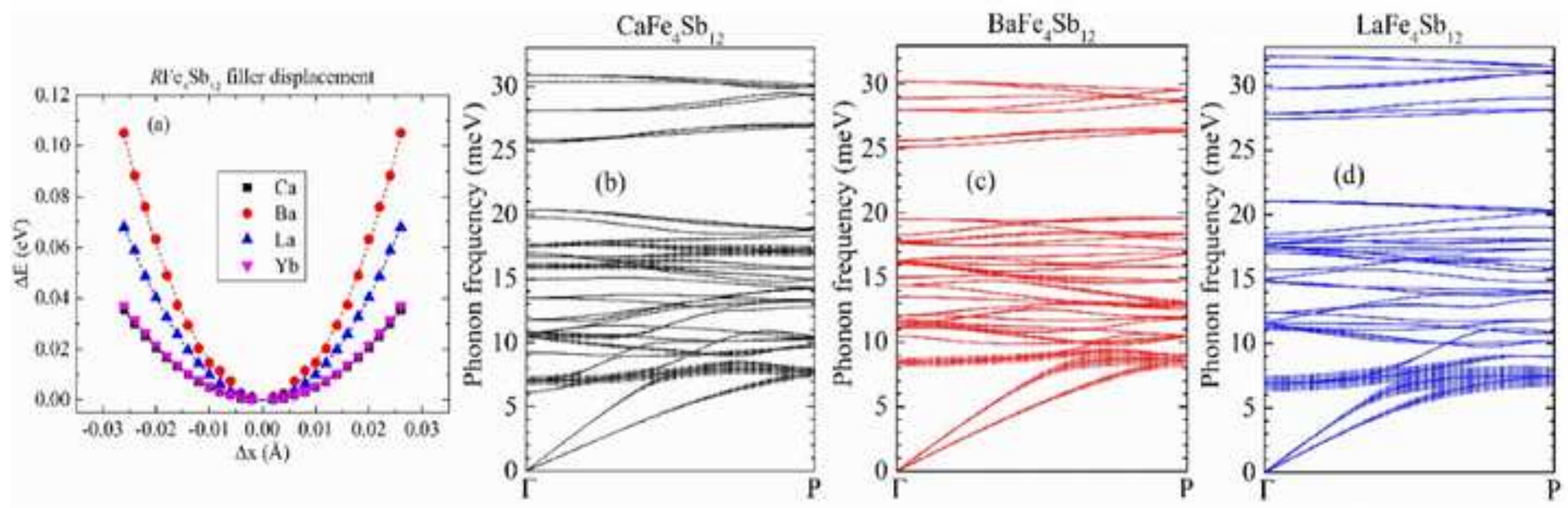


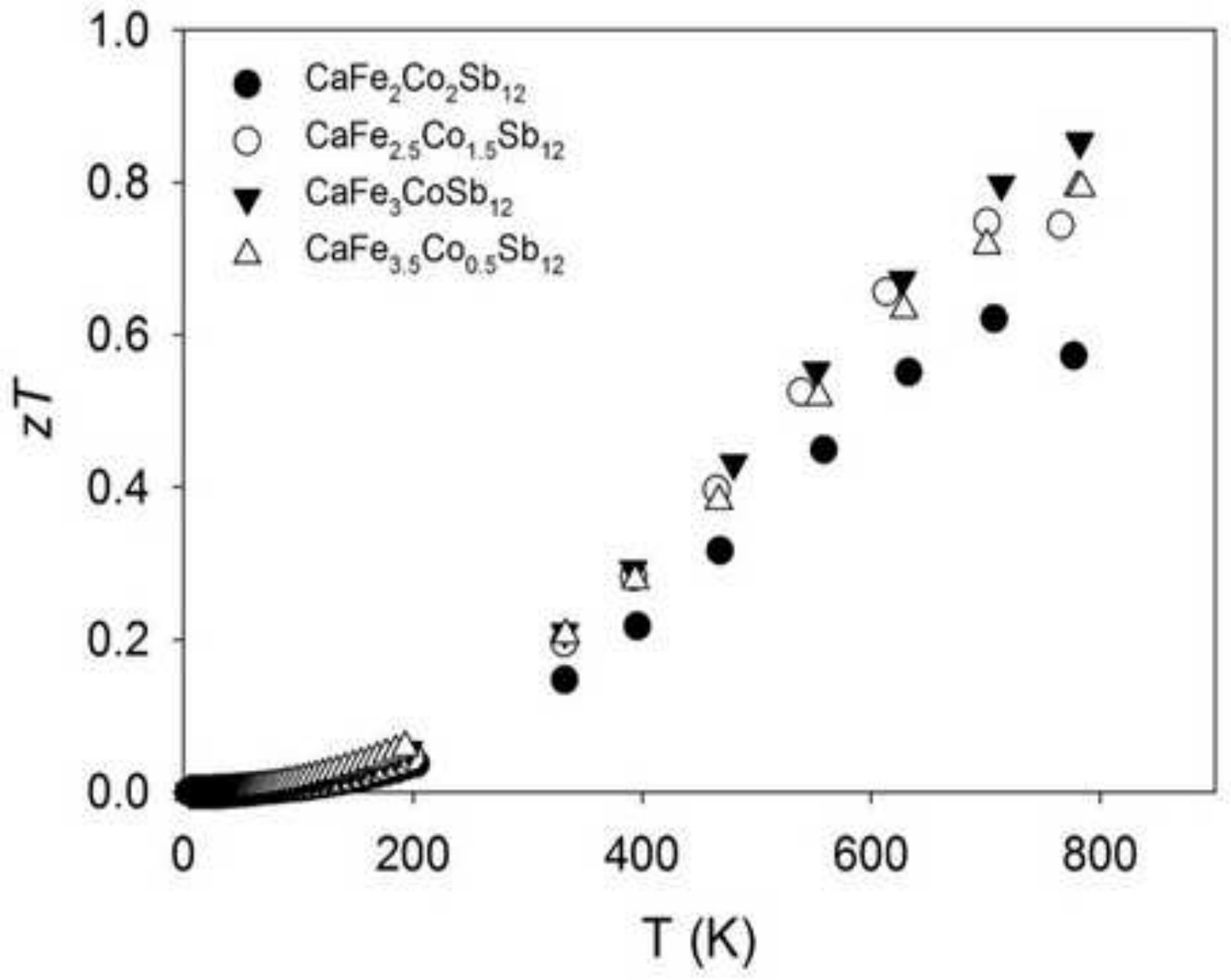



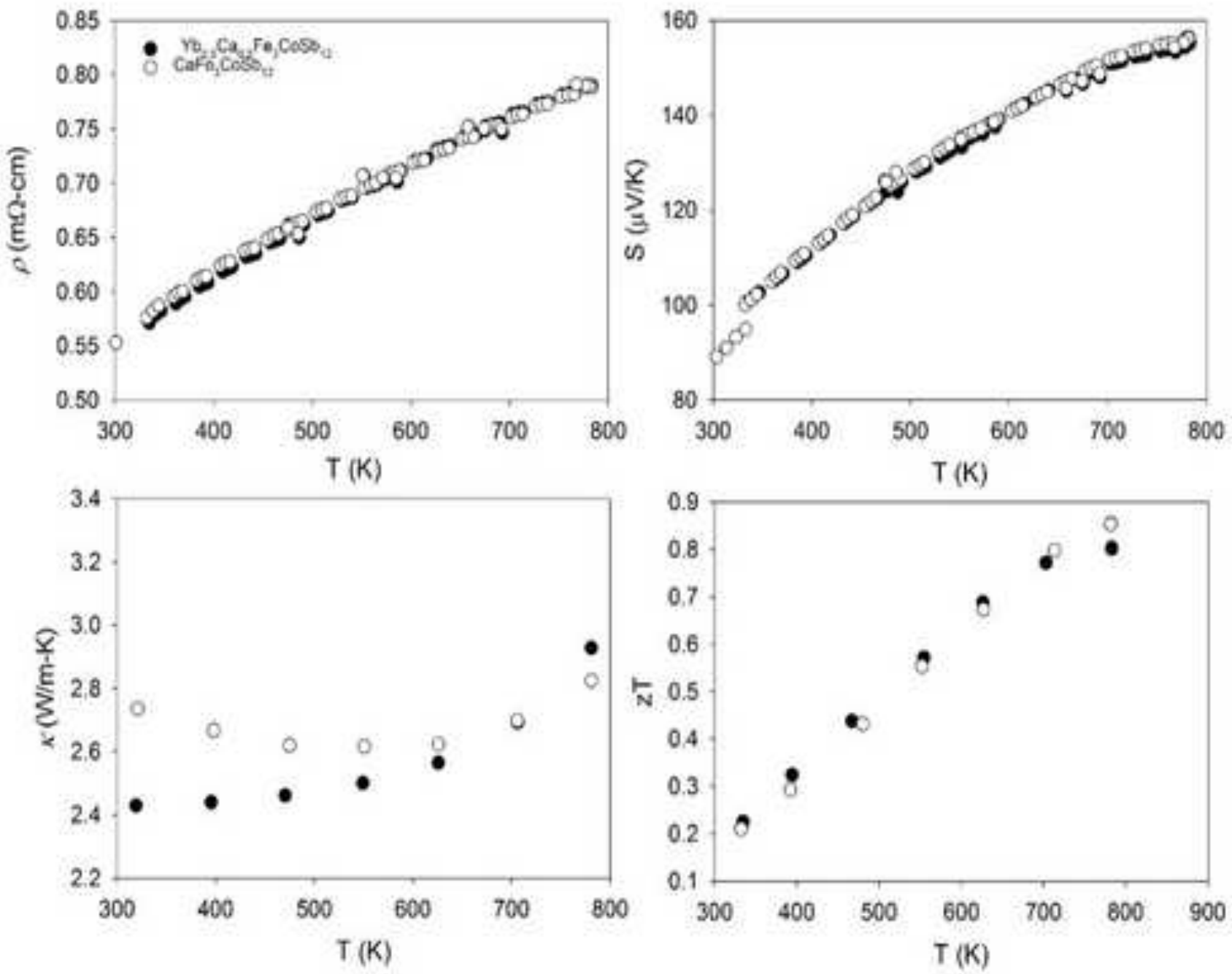